\title{
Simulation of Beam-beam Effects in Electron-positron Rings
}

\author{
Kohji HIRATA and Shuji MATSUMOTO \\ KEK, National Laboratory for High Energy Physics, Oho, Tsukuba, Ibaraki 305, Japan
}

\begin{abstract}
We examine the coherent beam-beam effects for the case of a vcry flat beam by multi particle tracking. On resonances, the coupling between coherent dipole mode and higher modes plays an important role.
\end{abstract}

\section{Introduction}

Multi-particle tracking(MPT) is a powerful tool to evaluate the beam-beam effects accurately[1,2]. In MPT, beam is represented by a cluster of particles and each particle motion is pursued numerically. The aim of this report is to examine the significant effects on the coherent dipole motion by MPT. To this end, it is valuable to consider the problem in a simplified situation: both beams are very flat. In this case, the dominant beambeam effect will appear in the vertical motion only. If we neglect the modulation on the vertical beam-beam force by the horizontal betatron motion, we can consider the beam-beam problem in one dimensional space. While this is a rather drastic simplification and an ideal mathematical limit, the result of this approach should be useful for further study on the beam-beam problem. In our approach, it is important that we evaluate the beambeam kick without any kind of assumption for beam distribution.

When estimating the beam-beam force in a tracking study, sometimes, the beam barycenter motoin is neglected (both beams are forced to be always in head-on collision)[1] or the bean envelope is approxinated as a Gaussian[2]. The former scheme artificially eliminates the contribution from the dipole mode and the latter from some part of other higher order modes. However this may give a great influence on the beam motion. In fact, as for the latter, the importance of non-Gaussian effect was already pointed out\{1]. In the present paper, we will see that we should also pay attention to the dipole motion.

MPT with these two schemes are less realistic than that with ours. We call the scheme of the former, MPT with Gaussian approximation, the Error-function Scheme (EFS) since the beam-beam force is described by the error function. As for the latter, MPT which does not include dipole mode, we call it the No-Dipole Scheme (NDS). Our scheme is called the Sorting Scheme (SS) since the beam-beam force calculation is executed based on the sorting algorithm.

\section{MPT in one dimensional space}

We consider a simple model ring that has only one interaction point(IP) and consists of only linear elements. The beam-beam kick at the IP is $\Delta Y_{1}=0$ and

$$
\Delta Y_{2}=-2 \pi^{3 / 2} \eta \int d Y_{1}^{*} \rho_{*}\left(Y_{1}^{*}\right) E\left(Y_{1}-Y_{1}^{*}\right)
$$

where $E(y)= \pm 1$ for $y \geq 0$, the quantity with * refers to the encountering bunch, $\rho$ is the distribution function and $\eta$ is the nominal beam-beam parameter. Here we use the canonical variables of an $e^{ \pm}, Y_{1}^{ \pm}=y_{ \pm} / \sigma_{O y}$ and $Y_{2}^{ \pm}=\beta_{0 y} y_{ \pm}^{\prime} / \sigma_{0 y}$, where $\sigma_{0 y}$ is the nominal vertical beam size and $\beta_{0 y}$ is the nominal betatron function at the IP. We can calculate the beam-beam force by counting the number of particles[3]. From Eq.(1), $\Delta Y_{2}=-2 \pi^{3 / 2} \eta\left(N_{*}^{u}-N_{*}^{d}\right) /\left(N_{*}^{u}+N_{*}^{d}\right)$, where $N^{u(d)}$ is the number of particles in the encountering bunch which are above (below) $Y_{1}$. Any approximation in evaluating the beam distribution $\rho$ is not necessary.

In the arc, the beams are transported with linear betatron motion perturbed by the effects of synchrotron radiation [4]: $\left(Y_{1}, Y_{2}\right)^{t}$ changes to

$$
\lambda\left(\begin{array}{cc}
\cos \mu & \sin \mu \\
-\sin \mu & \cos \mu
\end{array}\right)\left(\begin{array}{c}
Y_{1} \\
Y_{2}
\end{array}\right)+\sqrt{\left(1-\lambda^{2}\right)}\left(\begin{array}{c}
r_{1} \\
r_{2}
\end{array}\right)
$$

Here $\mu=2 \pi \nu$ ( $\nu$ being the tune), $\lambda=\exp (-1 / T)(T$ being the vertical damping time divided by the revolution time) and $r_{i}$ is a Gaussian random number with zero mean and the unit r.m.s.

We define the heam coherent, quantities as usual: for dipole modes, $\bar{Y}_{i}^{ \pm}=\left\langle Y_{i}^{ \pm}\right\rangle$and for quadurupoles, $M_{i j}^{ \pm}=$ $\left\langle\left(Y_{i}^{ \pm}-\bar{Y}_{i}^{ \pm}\right)\left(Y_{j}^{ \pm}-\bar{Y}_{j}^{ \pm}\right)\right\rangle$, where \langle\rangle means the average over the particles in a bunch and $i, j=1,2$.

\section{Result and Discussion}

We study the head-on collision only. The two beams have the same design orbit. We use a rather large beambeam parameter, $\eta=0.10$, in order to enhance the beam-beam effects

\subsection{Equilibrium behavior}

We employ following three average quantities to specify it: the average distance of two barycenters $D \equiv$ $\left\langle\left|\bar{Y}_{1}^{+}-\bar{Y}_{1}^{-}\right|\right\rangle_{a v}$, the average effective beam size $\Sigma \equiv$ 
$\left\langle\sqrt{M_{11}^{+}+M_{11}^{-}}\right\rangle_{a v}$ and the luminosity reduction factor:

$$
R=\left\langle\sqrt{\frac{2}{M_{11}^{+}+M_{11}^{-}}} \exp \left[-\frac{1}{2} \frac{\left(\bar{Y}_{1}^{+}-\bar{Y}_{1}^{-}\right)^{2}}{M_{11}^{+}+M_{11}^{-}}\right]\right\rangle_{a v} .
$$

Here \langle\rangle$_{\text {av }}$ means average over many turns. The real luminosity $L$ is $L_{0} \times R$ where $L_{0}$ is the nominal luminosity [5].

At first, we compare SS with EFS. The result is shown in Fig.1. Generally speaking, the equilibrium

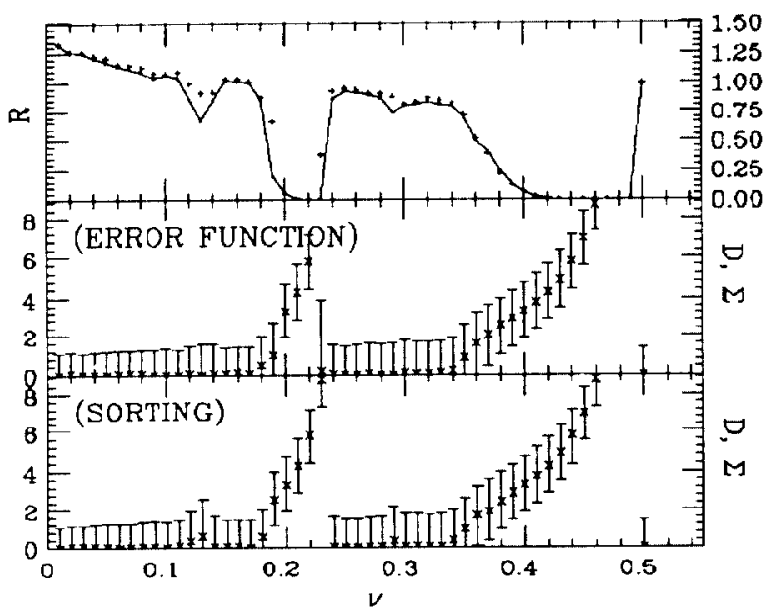

Figure 1: The tracking results of SS and FFS. (Top) the luminosity reduction factor $R$ by SS (line) and EFS $(+)$, (Middle) The barycenter difference $D(x)$ and the effective beam size $\Sigma$ (as error bars) by EFS. (Bottom) $D$ and $\Sigma$ in SS. Parameters: $\eta=0.10$ and $T=1000$. We started from $M=I$ and $Y_{1}^{+}-Y_{1}^{-}=0.0$ and tracked for 10000 turns. 1000 particles were used for each beam.

state predicted by EFS is very similar to the one by SS at almost all tunes. This is a surprising and interesting fact. As far as the equilibrium property is concerned, the Gaussian approximation for beam envelope seems to be good. There, however, are some exceptions.

We have a large dip in $R$ below $\nu=0.5$ (half-integer resonance). This is due to the coherent dipole instability $[6$, 7]. Another large dip is seen below $\nu=0.25$ (the fourth order resonance) both in SS and EFS. We naturally expect the quadrupole instability[6], however SS and EFS show the appearance of dipole oscillation instead of beam size enhancement. The particle distribution in phase space is shown in Fig.2. We have tiny dipole cxcitation at the sixth order and the third order resonances in SS, while it does not exist in EFS

Tostudy these properties of nonlinear resonances, we will observe what occurs on the beam motion in a way to the equilibrium
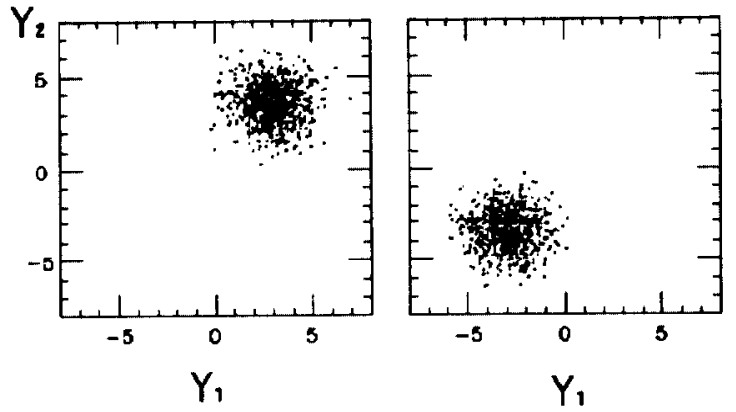

Figure 2: The phase space equilibrium distribution of $e^{+}$ and $e^{-}$bunches $\left(Y_{1}^{ \pm}, Y_{2}^{ \pm}\right)$on the fourth order resonance in SS. We have similar beam distribution in EFS. $\nu=$ 0.22 The system moves in period -4 .

\subsection{Transition properties}

Let us see the fourth order resonance first. See Fig. 3 . In

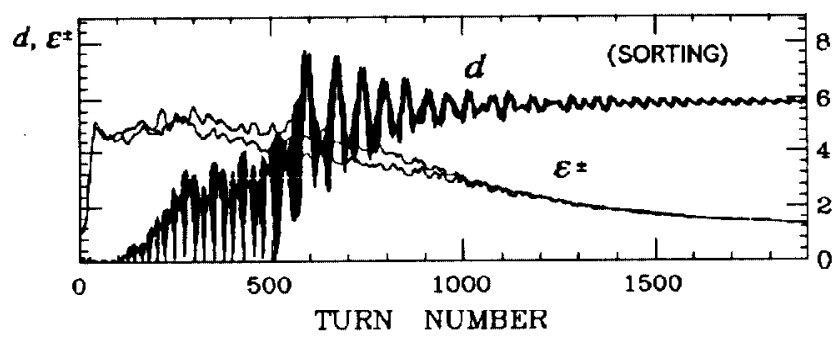

Figure 3: The changc of the barycenter difference, $d=$ $\left|Y^{-}-Y-\right|$ and $t$ wo beam emittances, $\epsilon^{ \pm}=\left(\operatorname{det} M^{ \pm}\right)^{1 / 2}$, by every turn. For (first) 2000 turns in SS. $\nu=0.22$.

SS, we have rapid growth of both beam sizes in the beginning. However, the dipole oscillation becomes prominent gradually and the beam sizes are slowly damped to the nominal one. The similar behavior was observed in EFS.

The same mechanism seems to work at the sixth order resonance (around $\nu=0.16$ ) but not apparently. We have beam size enhancement first, but the excitation of the dipole mode is extremely slow: we tracked for 90000 turus (for 90 damping time), but the system does not seem to be in equilibrium. The phase space distribution in SS is shown in Fig.4. It seems that $\eta=0.10$ is too small to excite a rapid and large dipole oscillation at the sixth order resonance. We observe a very small dipole excitation in EFS but it does not grow at all.

On the third order resonance, a similar mechanism seems to work. We found the dipole oscillation was excited only in SS. The origin of this excitation may attribute to the skewness of $\rho$, which makes the odd order 

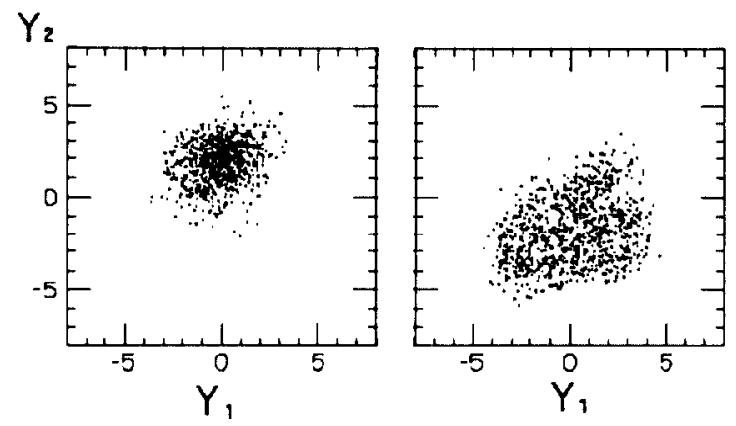

Figure 4: The particle distribution in phase space on the sixth order resonance after 90000 turns in SS. $\nu=0.13$. The system may not still be in the equilibrium. 'I'wo beam envelopes are asymmetric $\left(\left\langle M_{11}^{+} / M_{11}^{-}\right)_{a v}=2.76\right)$. Dipole mode is exited $(D=2.0)$.

components in beam-beam force with respect to $Y_{1}$ even with head-on collision. We found the flip-flop states in the equilibrium in SS only. In EFS, because of this assumption, the skewness is zero, so that the dipole excitation does not occur. On the contrary, in the collision with the primordial offset at IP, third order resonance can be excited even in EFS.

Above results indicate the importance of the coupling between dipole and higher order modes. In this sense, NDS is a special scheme since it assumes the existence of feed-back system that eliminates completely the beam dipole excitation. Especially, in the case where the dipole mode plays a dominant role, NDS will give a very different result. Let us see it.

\subsection{No-Dipole Scheme}

In NDS, a completely different motion appears on above nonlinear resonances, since the barycenter motion is completely eliminated before the two beams collide. See Fig. 5 and compare it with Fig.2. In NDS, each beam splits into two pieces in phase space. Notice that the beam sizes change by period -2 and out of phase with each other. The assumption of a very fast and powerful feed-back system makes this difference.

\section{Conclusion}

We have realize that the coupling of coherent dipole mode and some other modes is important in the beambeam dynamics. Its prominent effect was seen on the forth order resonance, where the quadrupole mode was unstable first, but the dipole node was excited gradually and finally beam separation took place. On the sixth order resonance, the same mechanism seems to work but not so obvious. Another example was seen at the third order resonance. It seems that a flip-flop phenomenon and a small dipole excitation coexist in the equilibrium.
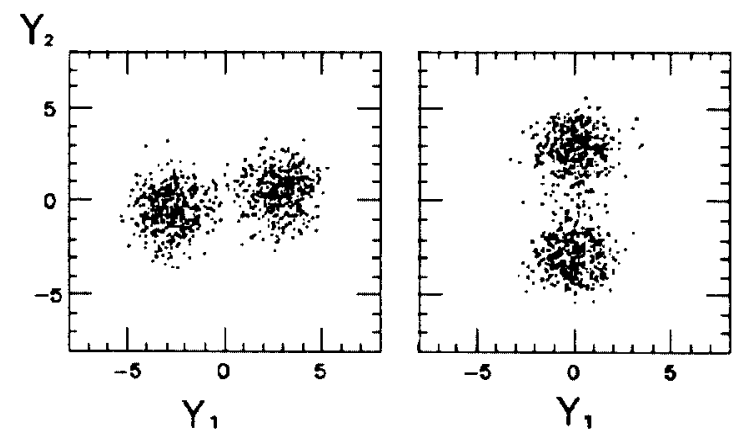

Figure 5: The equilibrium particle distribution in phase space in NDS. $\nu=0.22$. The system moves in period-4, but the distribution is drastically different from that of SS. See Fig.2.

As stressed in [1], we should not use any assumption in calculating the beam-beam force. This applies for the dipole mode. The NDS treats the very special situation: ring with an extremely fast feed-back system.

\section{References}

[1] S. Krishnagopal and R. Siemann, Phys.Rev.Lett., 67, 2461 (1991).

[2] A.Piwinski, in Proc. 11 th Int. Conf. on High Energy Accelerators, Genève-July 1980, 751 (Birkhäuser,1980); E.Keil, Nucl. Instrum. Methods 188, 9 (1981); S. Myers, Nucl. Instrum. Methods $211,263(1983)$.

[3] K. Hirata, KEK Preprint-92-83 (1992), to appear in Particle Accelerators.

[4] K. Hirata and F. Ruggiero, Particle Accelerators 28, 137 (1990).

[5] Strictly speaking, the calculation of luminosity (reduction factor) by this method is not correct for non-Gaussian beam.

[6] N. S. Dikanski and D. V. Pestrikov, Particle Accelerators 12, 27 (1982); A.W. Chao and R.D. Ruth, Particle Accelerators 16, 201 (1985); P. Zenkevich and K. Yokoya, KEK Preprint 92-116 (1992).

[7] K. Hirata and E. Keil, Nucl. Instrum. Methods A292, 156 (1990). 